\title{
Detection and Correction of Radiance Variations During Spectral Calibration in APEX
}

\author{
Michael Jehle, Andreas Hueni, Member, IEEE, Karim Lenhard, Andreas Baumgartner and Michael E. \\ Schaepman, Senior Member, IEEE
}

\begin{abstract}
The Airborne Prism EXperiment (APEX) is an imaging spectrometer developed by a joint Swiss-Belgian consortium composed of institutes (University of Zurich, Flemish Institute for Technological Research) and industries (RUAG, OIP, Netcetera), supported by the European Space Agency's PRODEX programme. APEX is designed to support the development of future space-borne Earth observation systems by simulating, calibrating or validating existing or planned optical satellite missions. Therefore, periodic extensive calibration of APEX is one major objective within the project. APEX calibration under laboratory conditions is done at its dedicated calibration and characterization facility at the DLR in Oberpfaffenhofen, Germany.

While environmental influences under laboratory conditions are reduced to a minimum, the effects of atmospheric absorption and the properties of the underlying calibration infrastructure may still influence the measurements and subsequently the accuracy of the sensor spectral response estimations. This paper presents an analysis of these effects and a method for their compensation. The correction approach is exemplarily demonstrated on actual measurements for the SWIR channel.
\end{abstract}

Index Terms- Airborne Prism Experiment, APEX, atmospheric absorption, calibration, measurement setup, radiance, spectral.

\section{INTRODUCTION}

$\mathrm{A}$ PEX_is a dispersive pushbroom imaging spectrometer that measures the solar reflected radiance in the wavelength range from $380 \mathrm{~nm}$ to $2500 \mathrm{~nm}$, using two spectrometer channels that share a common ground imaging optics. The ground sampling distance of the 1000 spatial across track pixels depends on the flight height in combination with the instruments Field of View (FOV) of $28^{\circ}$ and is approximately $1.75 \mathrm{~m}$ at $3500 \mathrm{~m}$ above ground level. Radiances are measured with up to 532 spectral bands. More than 330 spectral bands thereof are freely reconfigurable.

Table 1 shows the main properties of the two APEX imaging spectrometer channels.

Manuscript received xxx, 2014;

M. Jehle, A. Hueni and M. E. Schaepman are with the Remote Sensing Laboratories, Department of Geography, University of Zurich, ZH 8057 Switzerland (e-mail: michael.jehle@geo.uzh.ch).

K. Lenhard and A. Baumgartner are with the German Aerospace Center (DLR), Institute für Methodik der Fernerkundung (IMF), 82234 Oberpfaffenhofen, Germany.
TABLE 1: APEX MAIN PROPERTIES

\begin{tabular}{|l|l|l|}
\hline \multicolumn{2}{|l|}{ APEX Performance } \\
\hline \hline Spectral Performance & VNIR & SWIR \\
\hline Spectral Range & $372-1015 \mathrm{~nm}$ & $904-2508 \mathrm{~nm}$ \\
\hline Spectral Bands & $\begin{array}{l}\text { up to } 334, \\
\text { default: } 114\end{array}$ & 198 \\
\hline Spectral Sampling Interval & $0.45-7.5 \mathrm{~nm}$ & $5-10 \mathrm{~nm}$ \\
\hline Spectral Res. (FWHM) & $0.7-9.7 \mathrm{~nm}$ & $6.2-12 \mathrm{~nm}$ \\
\hline \hline Spatial Performance & (across track) \\
\hline Spatial Pixels & 1000 & \\
\hline FOV & $28^{\circ}$ & \\
\hline IFOV & $0.028^{\circ}(\sim 0.5 \mathrm{mrad})$ \\
\hline Spatial Sampling Interval & $1.75 \mathrm{~m} @ 3500 \mathrm{~m}$ AGL \\
\hline \hline Sensor Characteristics & VNIR & SWIR \\
\hline Type & CCD & CMOS \\
\hline Dynamic Range & 14 bit & 13 bit \\
\hline Pixel Size & $22.5 \mu \mathrm{m}$ by & $30 \mu \mathrm{m}$ by \\
\hline Smile & $22.5 \mu \mathrm{m}$ & $30 \mu \mathrm{m}$ \\
\hline Keystone (Frown) & average $<0.35$ pixel \\
\hline Co-Registration & average $<0.35$ pixel \\
\hline \hline & average $<0.55$ pixel \\
\hline \hline
\end{tabular}

The APEX calibration concepts aim for a complete sensor characterisation in terms of spectral, geometric and radiometric behaviour of the instrument. The instrument characterisation enables the full understanding of the sensor, and the basis for data calibration, traceably translating the measured raw data to physical units within the APEX Processing and Archiving Facility [1-5].

For a most accurate sensor characterization measurement uncertainties and their error propagation need to be known and if possible corrected [6], [7]. In this paper, we attempt to reduce potential sources of systematic errors in the determination of the spectral calibration of APEX caused by the measurement setup and environmental constraints. The presented correction approach can be used to compensate for these effects which are mostly due to the non-perfect measurement setup. 


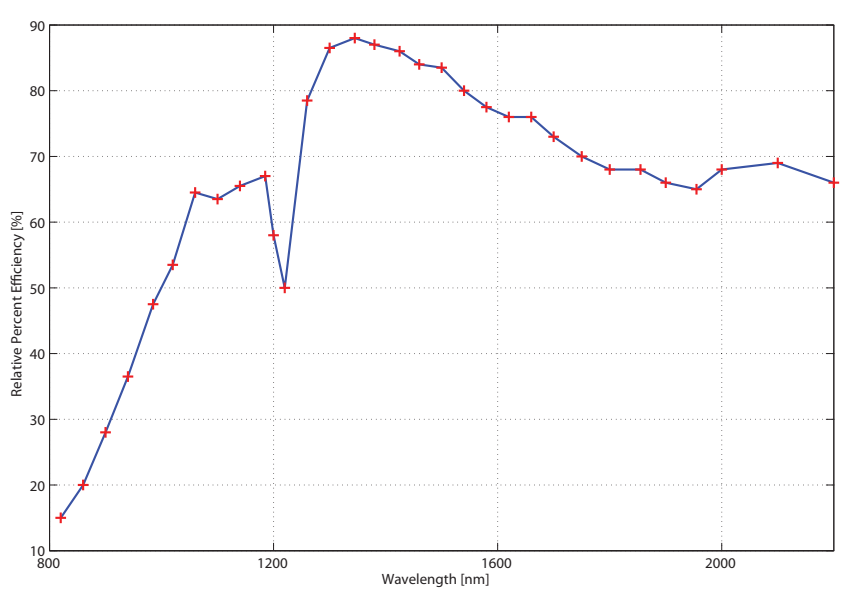

Fig. 1. Example of a monochromator grating efficiency provided by Newport, modified from [13]

\section{MEthodS}

\section{A. APEX Spectral Calibration}

The APEX spectral calibration setup includes a monochromator (Type: Oriel MS257, see [8]) that generates a narrow-band beam of light. The light is collimated and directed to a folding mirror reflecting the beam into the entrance aperture of APEX. A detailed description of the measurement setup is provided in [7]. The spectral response functions are determined for all spectral bands using a reference set of geometric pixels. Therefore the sensor aperture is illuminated only at defined angles. The complete spectral response for both channels (SWIR and VNIR detector) is subsequently estimated by fitting to the reference subset of each detector.

For the SWIR detector, for example, selected pixels are scanned spectrally in a window of 50 consecutive steps of 1 $\mathrm{nm}$ (scan window). This number of steps and bandwidth was found sufficient as the bandwidth of the SWIR spectral response is always below $\sim 12 \mathrm{~nm}$. The bandwidth of the monochromator depends on the slitwidth of the monochromator and the dispersion of the grating and is always below $1.2 \mathrm{~nm}$ for all gratings used.

The measured responses are usually well described by Gaussian functions [9]. By fitting Gaussians to the data points, the bandwidth, defined as the Full Width at Half Maximum (FWHM) and the centre wavelength (CWVL) positions can easily be extracted. This approach works well under the assumption that the spectral radiance reaching the detector changes only slowly over the scan window of $50 \mathrm{~nm}$.

\section{B. Detection and Correction of Radiance Variations During Spectral Calibration}

Higher level processing of spectral data requires highly accurate calibration and preprocessing for acceptable estimations of e.g., vertical column density (VCD) of atmospheric $\mathrm{NO}_{2}$ concentration [10] or chlorophyll fluorescence [11]. While the sensor instrument setup remains the same during laboratory calibration or during airborne

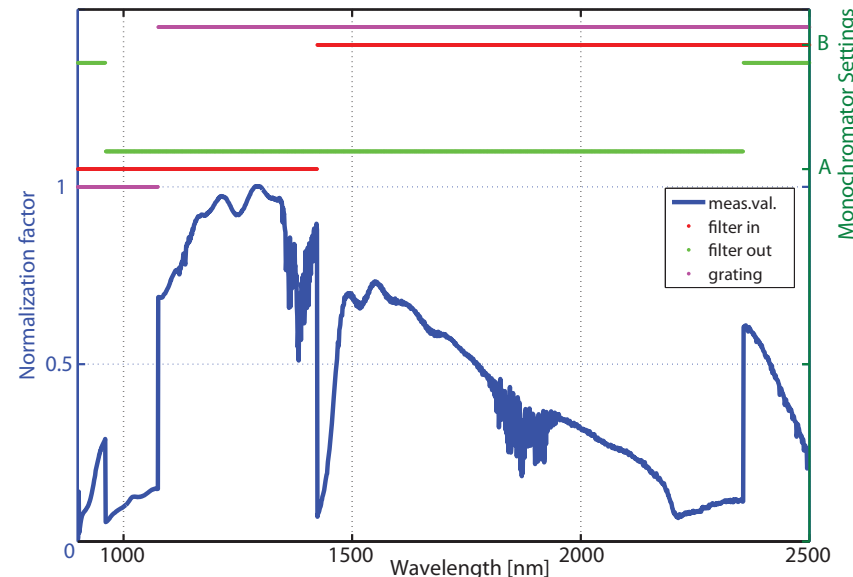

Fig. 2. Estimated normalization factor relative to maximum measured light level on the SWIR detector. The horizontal lines indicate the location of changes of the monochromator setup.

measurement campaigns, the measurement setup changes. Large variations in the following parameters of the measurement setup are expected to critically distort the atsensor radiance within the scan window and can strongly affect the performance and accuracy of retrieved remote sensing products:

- The spectral radiance emitted from the Quartz Tungsten Halogen (QTH) lamp [12]

- The transmission of the monochromator, mostly defined by the grating efficiency

- Atmospheric absorption (e.g. $\mathrm{H}_{2} \mathrm{O}, \mathrm{O}_{2}$ )

To accurately measure the response of the detector to incoming light, the effects of these parameters need to be quantified using dedicated measurements and afterwards corrected. A common irradiance distribution of a Quartz Tungsten lamp as it is used for the APEX spectral calibration can be seen from [8]. Its variation over the calibration wavelength range is comparably smooth and can be neglected within a scan window of $\sim 50 \mathrm{~nm}$.

Fig. 1 shows an example of a grating efficiency used for spectral calibration. Some gradients are large in comparison to those of the light source and can have a strong impact on the results of the spectral measurements. Effects within the APEX instrument are assumed to be independent from changes within the measurement setup.

\section{Measurements \& Correction Method}

The spectral response of the SWIR detector was measured at its centre spatial position using scan segments of $1 \mathrm{~nm}$ within the spectral range of 980 to $2500 \mathrm{~nm}$. One SWIR detector frame consists of 1000 by 198 pixels. A SWIR frame is first corrected by its dark current. Second, every pixel from each measurement frame needs to be normalized to the total amount of light impinging the detector. As the pixels are not yet radiometrically calibrated each pixel value is approximated by normalizing to the overall detected sum of the DN values. This normalization allows for a comparison of DN levels between subsequent frame acquisitions. Each frame 


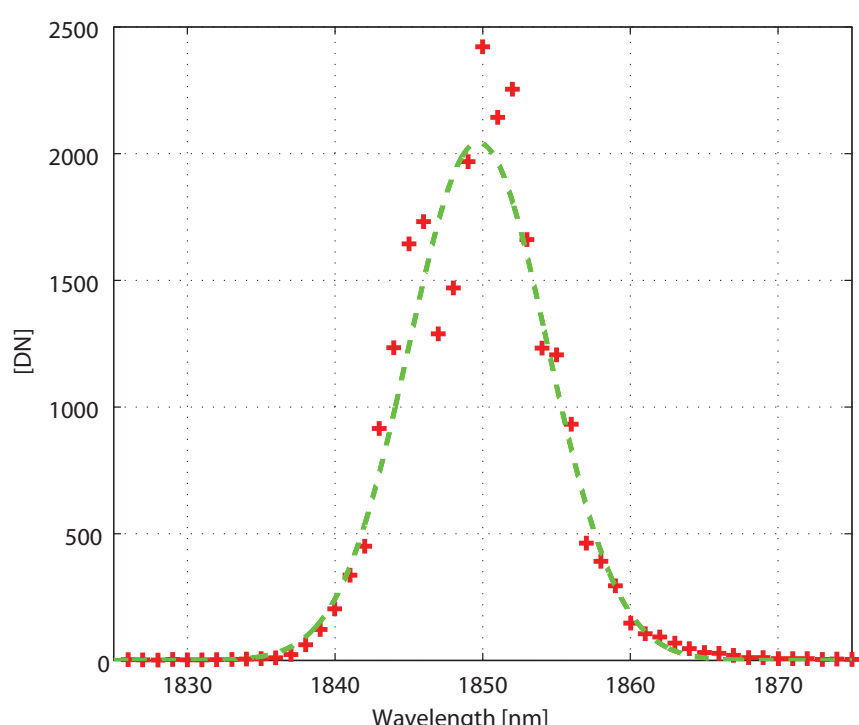

Fig. 3. Spectral response function without correction (CWVL: $1849.57 \mathrm{~nm}$, FWHM: $12.50 \mathrm{~nm}, \mathrm{R}^{2}$ : 0.9583).

is normalized to the maximum signal level observed within the complete scan window measurements. This normalization procedure corrects for unwanted changes in light intensity between the calibrating light source (QTH) and the detector.

The spectral response for a specific pixel on the detector is then estimated by fitting a Gaussian through the converted pixel's DN level.

Technically, the light level per frame $\left(D N_{\text {dect }}\right)$ is retrieved from a summation of every spectral ( $i$ ) and spatial $(j)$ pixel DN contribution over the whole detector (dect) using:

$$
D N_{d e c t}=\sum_{i=1}^{199} \sum_{j=1}^{1000} D N_{i j}
$$

Each measurement frame (FRME) is then normalized to the overall maximum of all frames within the complete scan window according to:

$$
\begin{aligned}
& F R M E_{\text {norm }}(k) \\
& =F R M E(k) \cdot \frac{D N_{\text {dect }}(k)}{\max \left\{D N_{\text {dect }}(1), D N_{\text {dect }}(2), \ldots D N_{\text {dect }}(n)\right\}},
\end{aligned}
$$

where $\mathrm{k}$ defines the frame numbers and $\operatorname{FRME}(1,2, \ldots . n)$ in this case is a cube consisting of 2500 APEX frames collected within the whole SWIR spectral range.

Figure 2 shows the normalization factor (second factor of multiplication in (2)) for each frame over the whole SWIR scan window. The normalization factor is plotted together with the status of the monochromator filter and grating settings. A change in its respective values corresponds to a filter or grating change at the position of its change. A weighted moving average filter adapted to the locations of the monochromator filter or grating changes was used to further reduce high frequency noise contributions which were introduced by positioning inaccuracies of the step motor of the

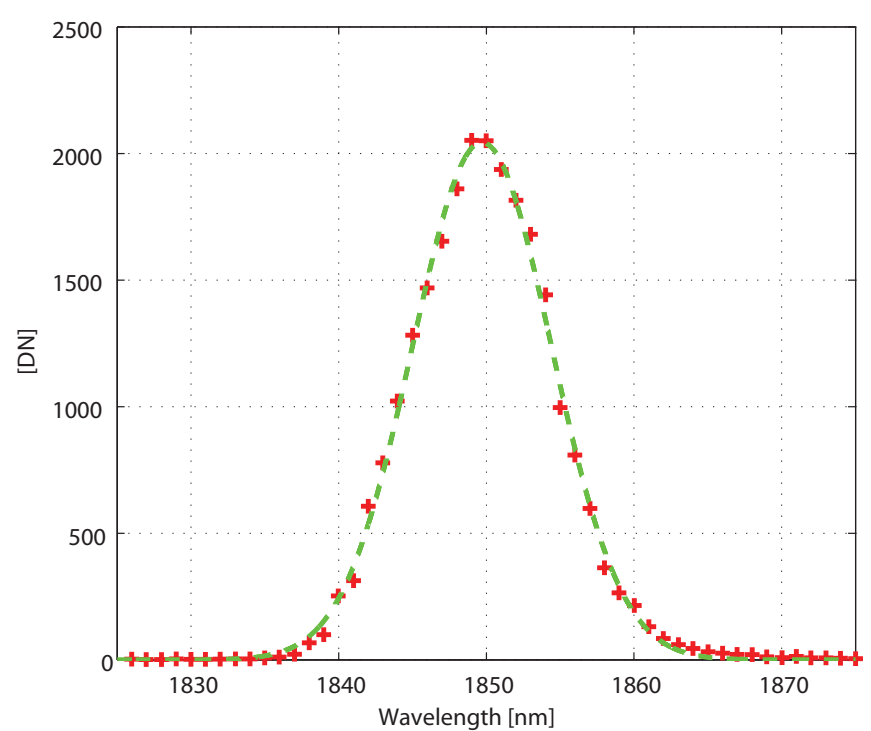

Fig. 4. Spectral response function after correction. For comparison the measurement points were multiplied by an average peak intensity (CWVL: 1849.59 nm, FWHM: 11.28 nm, $\mathrm{R}^{2}$ : 0.9972).

monchromator grating [14]. The spectral response function (SRF) can now be extracted for all pixel positions using:

$$
\begin{aligned}
& \operatorname{SRF}(k)=F R M E_{\text {norm }}(i, j, k) \\
& k=\Delta: \Delta+49
\end{aligned}
$$

where $\Delta$ defines the starting frame at which the scan (over a 50 steps of $1 \mathrm{~nm}$ window) for the SRF begins. From Fig. 2 it is obvious that the most critical impact on the spectral response estimation is expected around the wavelength of the atmospheric absorption at $1400 \mathrm{~nm}$ and $1850 \mathrm{~nm}$. Therefore, Figure 3 shows an example of an extracted SRF around a wavelength of $1850 \mathrm{~nm}$ while the correction algorithm was not applied to the measured frames. The red crosses indicate the measurement points and the green dashed line indicates the Gaussian fit function according to:

$$
\text { SRF } F_{\text {gaussfit }}=a \cdot e^{-\left(\frac{x-b}{c}\right)^{2}}
$$

where $a, b$ and $c$ are the fit-function dependent least-squares estimated coefficients through this points. Thus, the position of the value $x=b$ corresponds to the estimated CWVL for a pixel $(i, j)$. The FWHM corresponds to $\sqrt{2 \ln 2} \cdot c$ of the Gaussian fit function. Figure 4 shows the same example as in Fig. 3 except that the measured detector frames were denoised and normalized to the estimated overall maximum light level (according to (2)).

\section{RESULTS \& DISCUSSION}

The general shape of the curve progression in Fig. 2 shows that the light conditions under which the detector is illuminated change significantly over the detector's bandwidth. The continuous, wavelength dependent variations in Fig. 2 are mainly due to the individual spectral variation of the lamp irradiance, the monochromator transmission and the 
atmospheric absorption. Also the detector's quantum efficiency and its pixel non-uniformity contribute to these variations. As the quantum efficiency of the SWIR detector material has only very small variations over the investigated wavelength area (compared to the other effects) and the correction of the pixel non-uniformity would require iterative radiometric calibration the correction for these effects was not considered. The jumps at $955 \mathrm{~nm}, 1075 \mathrm{~nm}, 1425 \mathrm{~nm}$ and $2355 \mathrm{~nm}$ are caused by filter- and grating changes of the underlying monochromator measurement setup.

Besides this general trend the curve is then mostly characterized by its disturbances measured around the wavelengths at $\sim 1400 \mathrm{~nm}$ and $\sim 1850 \mathrm{~nm}$. These are a result of the atmospheric absorption in this region and are caused by the atmospheric $\mathrm{H}_{2} \mathrm{O}$.

From Fig. 2 it is obvious that the atmospheric influence is severe even under laboratory conditions and needs to be corrected for. Furthermore, $\mathrm{H}_{2} \mathrm{O}$ concentration can vary over time leading to an unpredictable impact of this effect. Such disturbances have a considerable impact on the progression of the spectral response function as the spectral radiance that reaches the detector can change significantly with every wavelength increment. A robust method for reducing this atmospheric effect is to assume a constant radiance at the detector over the whole scan window.

Fig. 3 and Fig. 4 show the response function before and after the correction for the atmospheric distortion at a wavelength of $1850 \mathrm{~nm}$. For visualisation reasons the normalized measurement points in Fig. 4 were multiplied by an average peak intensity measured during the scan window for this particular SRF. While in this example the FWHM change is $<1.3 \mathrm{~nm}$ and the center wavelength change is safely below $0.1 \mathrm{~nm}$, differences can significantly increase in regions where additional effects are present. A more accurate quantitative estimation of the effect is difficult as the changes additionally depend on the method used for fitting the measurement points (e.g. parameterization, weighting or order of Gaussian fit). From Fig. 2 it also becomes possible to estimate the measurement uncertainty if the measurements were left uncorrected. From an estimation of the relative changes of the correction factor between two measurement points the uncertainty around a wavelength of $1850 \mathrm{~nm}$ can for example easily be up to $500 \mathrm{DN}$. However, if the correction algorithm is applied the measurement uncertainties drop significantly. The remaining uncertainties are then mainly due to the detector noise level ( $\sim 3$ to $10 \mathrm{DN})$ which is partly depending on the amount of light on the detector (shot noise) and the wavelength uncertainty generated by the monochromator (+/-0.1nm [8]). Therefore, in extreme cases the measurement uncertainty still might be up to $\sim 100 \mathrm{DN}$.

\section{CONCLUSIONS}

The measurements in Fig. 2, Fig. 3 and Fig. 4 show that atmospheric absorption can significantly distort spectral calibration measurements around $1400 \mathrm{~nm}$ and $1850 \mathrm{~nm}$ although the length of the light path through the atmosphere is only around $\sim 2 \mathrm{~m}$. Additionally, the monochromator grating efficiency can cause notable changes in at-sensor radiances while the variations introduced by the halogen lamp can be neglected within the scan window of $50 \mathrm{~nm}$ for the estimation of a SRF in the SWIR region. The corrections were demonstrated for the SWIR channel only but can also be applied in the same way for the VNIR channel.

The results indicate that the laboratory based estimation of the spectral response of the detector is possible even in regions of strong atmospheric absorption and during variations of the monochromator grating efficiency or even while changing the monochromator setup (different filter, type of grating). The demonstrated correction approach is able to compensate for atmospheric effects and undesired effects caused by the nonperfect measurement setup. An additional radiometric correction of the involved pixels would further improve the results. The method might also allow for estimations of the current atmospheric state by evaluating the degree of correction and normalization that has to be applied.

\section{REFERENCES}

[1] A. Hueni, J. Biesemans, K. Meuleman, F. Dell'Endice, D. Schläpfer, S. Adriaensen, S. Kempenaers, D. Odermatt, M. Kneubuehler, J. Nieke, and K. Itten, "Structure, Components and Interfaces of the Airborne Prism Experiment (APEX) Processing and Archiving Facility," IEEE Transactions on Geoscience and Remote Sensing, vol. 47, pp. 29-43, 2009.

[2] A. Hueni, K. Lenhard, A. Baumgartner, and M. Schaepman, "The APEX (Airborne Prism Experiment - Imaging Spectrometer) Calibration Information System," IEEE Transactions on Geoscience and Remote Sensing, vol. epub ahead of print, 2013.

[3] M. E. Schaepman, M. Jehle, A. Hueni, K. Meuleman, The APEX Team, and K. I. Itten, "The 4th generation imaging spectrometer APEX and its application in Earth observation," IEEE Transactions on Geoscience and Remote Sensing, in preparation.

[4] www.apex-esa.org

[5] M. Jehle, M. E. Schaepman, A. Hueni, A. Damm, P. D'Odorico, J. Weyermann, M. Kneubühler, and K. Meuleman, "APEX - current status, performance and validation concept," In, Sensors, 2010 IEEE (pp. 533537), Waikoloa, HI, USA, 2010

[6] Schaepman, M.E., \& Dangel, S. (2000). Solid laboratory calibration of a nonimaging spectroradiometer. Applied Optics, 39, 3754-3764

[7] P. Gege, J. Fries, P. Haschberger, P. Schötz, H. Schwarzer, P. Strobl, B. Suhr, G. Ulbrich, and W. J. Vreeling, "Calibration facility for airborne imaging spectrometers," ISPRS Journal of Photogrammetry \& Remote Sensing, vol. 64, pp. 387-397, 2009.

[8] Newport, 2007a: Oriel MS257TM 1/4 m monochromator and imaging spectrograph. http://assets.newport.com/pdfs/g5478.pdf (last visited 27.08.13)

[9] F. Dell'Endice, J. Nieke, D. Schlaepfer, A. Hueni, and K. Itten, "Automatic Calibration and Correction Scheme for APEX (Airborne Prism Experiment spectrometer)," in Proceedings of 5th EARSeL Workshop on Imaging Spectroscopy, Bruges, Belgium, 2007.

[10] C. Popp, D. Brunner, A. Damm, M. Van Roozendael, C. Fayt \& B. Buchmann (2012), "High resolution $\mathrm{NO}_{2}$ remote sensing from the Airborne Prism EXperiment (APEX) imaging spectrometer". Atmospheric Measurement Techniques, 5, 2211-2225.

[11] A. Damm, A. Erler, W. Hillen, M. Meroni, M.E. Schaepman, W. Verhoef \& U. Rascher (2011), "Modeling the impact of spectral sensor configurations on the FLD retrieval accuracy of sun-induced chlorophyll fluorescence". Remote Sensing of Environment, 115, 1882-1892.

[12] Oriel, "Oriel Product Training - Spectral Irradiance", http://www.newport.com/Quarz-Wolfram-Halogen-

Lampen/378263/1031/info.aspx\#tab_Literature Light_Sources.pdf, 39p., last visited: 04.04.2014

[13] Personal communication with Newport

[14] Personal communication with DLR 